\title{
Una Mirada Reflexiva sobre el Trasplante de Útero como un Novedoso Desafío del Derecho en la Legislación Colombiana
}

\author{
A Reflective Look at Uterus Transplantation as a Novel Challenge of Law in Colombian \\ Legislation \\ a Ángel David Paternina Petro ${ }^{57}$

\begin{abstract}
a angeldavid-1997@hotmail.com "Semillero Justicia, Política y Derecho", “Grupo de Investigaciones Sociales y Jurídicas”, Programa de Derecho, Fundación Universitaria Tecnológico Comfenalco. Cartagena, Colombia.
\end{abstract}

\begin{abstract}
Forma de Citar: A.D. Paternina-Petro, "Una Mirada Reflexiva sobre el Trasplante de Útero como un Novedoso Desafío del Derecho en la Legislación Colombiana", Rev. Saberes, Vol. 13, No. 02, pp. 81 - 87, 2020.
\end{abstract}

\begin{abstract}
Recibido: marzo 24 de 2020 Evaluación: mayo 28 de 2020 Aceptado: junio 30 de 2020 DOI: https://doi.org/10.25213/1794-4384/1302.0011
\end{abstract}
\section{Resumen}

El presente proyecto de investigación en curso tiene como objetivo analizar los fundamentos legales, jurisprudenciales o de principios jurídicos, que permitan el trasplante de útero en el ordenamiento jurídico colombiano, por ser un novedoso desafío para el derecho en nuestro país. Se analizaron los casos más significativos a nivel internacional de este tipo de prácticas médicoquirúrgicas (tres casos), lo que nos permitió observar los tipos de trasplante de útero, así como su posible uso, la viabilidad médica y jurídica y la aplicación e impacto en Colombia. En relación con lo investigado, se demuestra la viabilidad de esta práctica médica en nuestro país, con unas reglas especiales para cada caso, dependiendo el tipo de donante ya sea donante vivo o donante fallecido.

\section{Palabras Clave}

Componentes anatómicos, donante fallecido, donante vivo, trasplante de útero, reproducción asistida.

\section{Abstract}

The current research project aims to analyze the legal, jurisprudential or principled bases that allow uterine transplantation in the Colombian legal system, for being a new challenge to the law in our country. The most significant cases of this type of medical-surgical practices were analyzed at the international level (three cases), which allowed us to observe the types of uterine transplantation, its possible use, application and impact in Colombia. In relation to the investigation, the viability of this medical practice is demonstrated in our country, with special rules for each case, depending on the type of donor.

\section{Keywords}

Anatomical components, assisted reproduction, deceased donor, living donor, transplant of uterus.

\section{Introducción}

Desde el transcurso de la civilización y dado el desarrollo constante de la medicina, procedimientos que hace unas décadas eran impensables, en la actualidad son posibles; gracias a estos avances médico-quirúrgicos es viable hoy día salvar la vida de miles de personas en todo el planeta. Sin embargo, existen otros tipos de prácticas médicas, que si bien no son vitales para los seres humanos, si tienen un gran impacto en el proyecto de vida de cada uno de los individuos de la sociedad actual, por su incidencia a nivel social, psicológico, moral y en especial en el aspecto

\footnotetext{
* Autor para correspondencia: correo electrónico angeldavid-1997@hotmail.com 
familiar, toda vez que se traduce en una forma especial de conformación de familia $y$ en prolongación de la estirpe.

Dentro de los últimos avances quirúrgicos encontramos el trasplante de útero o trasplante uterino, que es un tipo de intervención donde se trasplanta el útero de una donante viva o fallecida, a una mujer que ha nacido sin útero o que lo ha perdido por alguna causa médica (Carmona, 2016). Siendo este un tipo de tratamiento complejo, que tiene como objeto la implantación del órgano donde se desarrolla y aloja el feto durante la etapa de gestación; cuya finalidad específica consiste en recuperar la fertilidad en la mujer $y$ en consecuencia la posibilidad practica de conformar familia.

Esta investigación en curso, tiene como objetivo general analizar los fundamentos legales, jurisprudenciales y de principios jurídicos que permitan el trasplante de útero en el ordenamiento jurídico colombiano, y como objetivos específicos se plantearon los siguientes: el primero consiste en identificar los tipos o formas de trasplante de útero en Colombia. El segundo radica en realizar un análisis de la casuística internacional sobre el trasplante de útero y un análisis de su incidencia en Colombia; y como tercer objetivo se plantea observar el impacto del trasplante de útero en las técnicas de reproducción humana asistida.

\section{Planteamiento del Problema}

El presente proyecto de investigación en curso tiene como pregunta problema la siguiente: ¿Existen fundamentos legales, jurisprudenciales o de principios jurídicos que permitan el trasplante de útero como novedoso desafio en el ordenamiento jurídico colombiano?

\section{Metodología}

La presente investigación en curso, es de enfoque cualitativo, de tipo dogmático y documental; su eje central gira en torno al análisis legal y casuístico del trasplante de útero en el ordenamiento jurídico colombiano. En este caso, de los tipos de trasplante uterino, su posible uso e implicación legal en nuestro país. Es de enfoque cualitativo, porque se basa en parámetros de análisis jurídicos con la finalidad de describir, establecer y sintetizar el nuevo fenómeno médico-quirúrgico del trasplante de útero. El método de investigación utilizado es el inductivo, bajo la modalidad de investigación documental. De igual forma acudimos a fuentes y técnicas de recolección de información; primarias y secundarias, tales como documentaciones oficiales, revistas científicas, investigaciones, entre otras.

\section{Avances, Resultados y Discusiones}

Desde hace más de tres décadas la medicina ha buscado una nueva alternativa para aquellas personas que sufren de infertilidad absoluta por factor uterino; siendo el trasplante de útero en la actualidad, aquella posibilidad terapéutica para restablecer la fertilidad en aquellas mujeres que por circunstancias de la vida no tienen un útero funcional (Correa, 2008).

El marco normativo que rige la donación y trasplante de componentes anatómicos ${ }^{58}$ es amplio y diverso, entre ellos encontramos el Decreto 2493 de 2004, que tiene como objeto regular la obtención, donación, preservación, almacenamiento, trasporte, destino y disposición final de componentes anatómicos y los procedimientos de trasplante o implante de los mismos en seres humanos. Este decreto en su Capítulo I, artículo $2^{\circ}$ que hace referencia a Definiciones, precisa que el trasplante es aquella: 'Utilización terapéutica de los órganos o tejidos humanos que consiste en la sustitución de un órgano o tejido enfermo, o su función, por otro sano procedente de un donante vivo o de un donante fallecido". Vislumbrando así, la posibilidad de dos tipos de donantes de componentes anatómicos en Colombia, la proveniente de un donante vivo y la procedente de un donante fallecido.

Con respecto a la primera, establece que: ' $E S$ aquella persona que conoce con certeza la totalidad de los riesgos que pueden generarse dentro del procedimiento y que cumpliendo los

\footnotetext{
58 Según el Decreto 2493 de 2004 en su artículo $2^{\circ}$, los componentes anatómicos 'Son los órganos, tejidos, células y en general todas las partes vivas que constituyen el organismo humano'.
} 
requisitos establecidos... efectúa la donación en vida de aquellos órganos o parte de ellos... ". Para este tipo de donantes, el decreto señala que debe ser una persona mayor de edad, que tenga un buen estado de salud, tenga plenas facultades mentales y se le haya advertido sobre la imposibilidad de conocer los riesgos y consecuencias que puedan ocurrir durante el procedimiento de extracción del órgano objeto del trasplante. Por su parte, la procedente de donante fallecido señala que: ' $E S$ aquel que ha fallecido bien sea por muerte encefálica $^{59}$ o por cese irreversible de las funciones cardiorrespiratorias y a quien se le pretende extraer componentes anatómicos con fines de trasplantes o implantes". En este tipo de donación, se establece que se deben realizar procedimientos de mantenimiento por medios artificiales al donante fallecido, a fin de que se garantice la viabilidad de los órganos destinados para el trasplante. De igual forma, la Ley 1805 de $2016^{60}$, modifica la presunción legal en materia donación de componentes anatómicos que tiene toda persona, después de haber ocurrido su deceso y para ello señala que la extracción no puede interferir con la práctica de necropsias y sus fines. Teniendo en cuenta lo anterior, se puede evidenciar que en nuestro país es permitido y es legal este tipo de prácticas médicas, lo cual favorecería la posible utilización del nuevo desafío médico-quirúrgico como lo es el trasplante de uterino, siempre y cuando se cumplan con los lineamientos establecidos en materia trasplante de componentes anatómicos y las reglas concernientes a la conservación y extracción, dependiendo el tipo de donante.

Antes de proceder a analizar los casos más representativos de este tipo de procedimientos a nivel internacional, es menester señalar que los antecedes históricos de esta práctica se remontan al año 2000, cuando en Arabia Saudita se realizó el primer trasplante de útero, el cual tuvo que ser removido tres meses después por problemas de coagulación en la sangre. Posteriormente en Turquía se realizó un segundo procedimiento

\footnotetext{
59 Señala el Decreto 2493 de 2004, que la muerte encefálica: ' $E s$ el hecho biológico que se produce en una persona cuando en forma irreversible se presenta en ella ausencia de las funciones del tallo encefálico, comprobadas por examen clínico".

${ }^{60}$ Por medio del cual se modifican la Ley 73 de 1988 y la Ley 919 de 2004 en materia de donación de componentes anatómicos y de dictan otras disposiciones.
}

uterino proveniente de una donante fallecida, donde se logró un embarazo en el año 2013, pero que tuvo que ser interrumpido porque no se detectó latido alguno en el feto (Diaz-García, et al., 2011). Dicho lo anterior, en este punto procederemos a analizar los casos más representativos de este tipo de prácticas médicas a nivel internacional; entre ellos, encontramos los siguientes:

\section{- Caso Suecia: donante vivo}

Una mujer de 36 años se convirtió en la primera del mundo en dar a luz tras un trasplante de útero. La receptora fue una de nueve mujeres que recibieron el trasplante del órgano en el 2013 y padecía de un trastorno genético por el cual nació sin útero. La donante fue una amiga de la familia de 61 años que había llegado a la menopausia siete años antes. Un año después del procedimiento trasfirieron un único embrión y tres meses después la mujer estaba embarazada. Durante la etapa de gestación la mujer presentó tres episodios de rechazo, pero los mismos fueron paliados con tratamientos inmunosupresores. El bebé ha nacido sano, en la semana 31 de gestación, con un peso total de 1.775 gramos, y mediante una cesárea. En: elpais.com [En línea]. 4 de octubre de 2014. [Consultado 17 de marzo de 2019]. Disponible en: https:/elpais.com/elpais/2014/10/04/ciencia/1412 406310 537420.html.

Como pudimos observar, el útero trasplantado provenía de una mujer mayor de edad, de 61 años, es decir de una donante viva. Si este caso hubiera ocurrido en nuestro país, a ambas pacientes se le debieron comunicar los posibles riesgos y consecuencias que podría tener este procedimiento quirúrgico a nivel social, familiar, personal y profesional. Ahora bien, si analizamos con profundidad el decreto 2493 de 2004, a la persona donante $^{61}$ y la receptora ${ }^{62}$, se le deberían haber realizado obligatoriamente por parte de los bancos de tejidos y las instituciones prestadoras de servicio a la salud, una serie de exámenes tales como: prueba de determinación de grupo sanguíneo, de sífilis, exámenes de síndrome de

\footnotetext{
${ }^{61}$ El decreto 2493 de 2004 reza en su artículo 2, que donante: ''Es la persona a la que durante su vida o después de su muerte, por su expresa voluntad o por la de sus deudos, se le extraen componentes anatómicos con el fin de utilizarlos para trasplante o implante en otra persona, con objetivos terapéuticos".

${ }^{62}$ Ibídem, señala que el receptor: 'Es la persona en cuyo cuerpo se trasplantan o implantan componentes anatómicos".
}

Página | 83 
inmunodeficiencia humana $\mathrm{VIH}$, prueba de hepatitis B y C, entre otras; a fin de que el trasplante de útero se realice con éxito y tenga menos probabilidad de rechazar el órgano.

Analizado el caso anterior proveniente de una donante viva, procederemos a estudiar uno de los casos más representativos a nivel internacional y el primero de donante fallecido que se ha realizado en el mundo. El cual se hará mención a continuación:

\section{Caso Brasil: donante fallecido.}

Los trasplantes de útero de donantes fallecidos son factibles. En Brasil nació el primer bebé el pasado 15 de diciembre de 2017 con esta técnica. La receptora fue una paciente de 32 años con infertilidad uterina. La cirugía tuvo lugar en septiembre de 2016. La donante tenía 45 años y murió de hemorragia subaracnoidea El útero fue extraído y trasplantado a la receptora en una cirugía que duro 10,5 horas. Diez días después de la implantación, se confirmó que la receptora estaba embarazada. La niña nació por cesárea a las 35 semanas, y pesaba 2.550 g. El útero trasplantado fue extirpado durante la cesárea y no mostró anomalías. En: Semana.com [En línea]. 5 de diciembre de 2018. [Consultado 17 de marzo de 2019]. Disponible en: https://www.elespectador.com/noticias/ciencia/na ce-el-primer-bebe-tras-un-trasplante-de-utero-deuna-donante-fallecida-articulo-827435.

El caso anterior es un hito para la medicina, debido a que por primera vez en toda la historia de la humanidad se logra realizar un trasplante de útero proveniente de una donante fallecida; y que como consecuencia de ello, naciera eventualmente una niña fruto de esta nueva técnica médicoquirúrgica. De igual forma pudimos observar que después de ocurrir el nacimiento de la menor, se procedió a la extirpación del órgano, por lo que podemos decir que el trasplante de útero se realizó con la finalidad de recuperar la fertilidad en el órgano reproductor de la mujer y concebir vida en él.

Dicho lo anterior, si este caso hubiera ocurrido en nuestro país, a la donante y receptora se le aplicarían las mismas reglas concernientes a la práctica de exámenes y pruebas del donante vivo, pero con la diferencia de que a la donante cadavérica, se le deben realizar los procedimientos de mantenimiento y sostenimiento a través de medios artificiales, con el fin de mantener la viabilidad de los componentes anatómicos que están destinados al procedimiento de trasplante y mirar si fue una donación consentida por ella en vida o producto de la aplicación de la presunción de donación, a la cual hoy en día no se podría oponer su familia, de acuerdo con la ley 1805 de 2016.

Es por ello que, el trasplante de útero representa para aquellas mujeres que sufren de infertilidadpor factor uterino- la posibilidad de contar con un tipo de tratamiento médico que tiene por objeto recuperar la fertilidad, y lograr un hijo biológico, toda vez que estas tenían como únicas opciones, la maternidad subrogada o la adopción, generando la primera de ellas una serie de complicaciones a nivel emocional, ético, moral, pero sobre todo legales, por la ausencia de legislación que regule esta práctica en Colombia. Siendo esta la oportunidad de generar un vínculo genético entre el niño y la madre; y de experimentar algunas sensaciones físicas y emocionales durante la etapa del embarazo. Y la segunda opción, por el largo tiempo que llega a tener un proceso de adopción en nuestro país.

Corolario a lo anterior y por la complejidad de esta nueva clase de procedimiento, autores como Ciamatori (2006), nos plantean observar los riesgos del trasplante desde el punto de vista, de la donante, la receptora y el niño por nacer. En el primer caso, se debe analizar desde dos perspectivas, la de donante cadavérico y donante de vivo. Para el primero señala que su objetivo solo se justifica en recuperar la función fisiológica básica necesaria para la vida, esto basado en la teoría ética del rescate de órganos. En el caso de donante vivo, los riesgos deben ser equiparados a los de una histerectomía, pero con un mayor grado de complejidad, por la necesidad de preservar el órgano trasplantado. En la receptora, se deben mirar las posibilidades médico-clínicas para trasplantar el útero y la capacidad psicológica para mantener órgano. Y desde el punto de vista del niño que está por nacer, las situaciones de pesos bajos y partos prematuros que puedan presentar.

Simultáneamente con los riesgos anteriores, se debe observar cada caso en específico, porque el procedimiento se encuentra aún en fase experimental, por lo que podría fallar y desencadenar en todo tipo de infecciones que colocarían en peligro la vida de las pacientes. Además de ello, como todo tipo de trasplante se

Página | 84 
pueden presentar situaciones de rechazo del órgano trasplantado, inmunosupresión por los medicamentos que injieren las pacientes $y$ adicional a ello, el riesgo que tiene la donante viva al momento de realizar el procedimiento de la extracción del útero. Es por estas razones que los médicos recomiendan proceder a la extirpación del órgano, después de conseguir su finalidad, como se pudo observar que se realizó en uno de los casos analizados.

Es sabido, que a partir de la expedición de la Carta Política colombiana de 1991 se establece la posibilidad de existencia de hijos a través de las técnicas de reproducción humana asistida, toda vez que el artículo 42 inciso 5 manifiesta que 'los hijos habidos en el matrimonio o fuera de él, adoptados o procreados naturalmente o con asistencia cientifica, tienen iguales derechos y deberes. La ley reglamentara la progenitura responsable"' (Moadie, 2015). Siendo las técnicas de reproducción humana asistida, el 'conjunto de métodos biomédicos, que conducen a facilitar o substituir, a los procesos biológicos naturales que se desarrollan durante la procreación humana" (Santamaría, 2000).

Si bien contamos con un marco normativo que regula el trasplante de componentes anatómicos en Colombia, lo que favorecería la implementación del procedimiento en nuestro país; la realización de este tipo de prácticas médicas podría tardar, porque se deben analizar las circunstancias técnicas, humanas y profesionales que se necesitan para su viabilidad. Aun así, se evidencia el poco interés por parte de las entidades prestadoras del servicio a la salud de trasplantar órganos reproductivos.

Tenemos que destacar el hecho de que en los últimos años, más de 38 trasplantes de útero se han realizado en todo el mundo, pero de todos los casos presentados solo 11 han llegado a términos exitosos, por lo que en los próximos años estaríamos frente a un aumento de este tipo de procedimientos, convirtiéndose en una práctica habitual que por no estar regulada tendría precios elevados, impidiendo que aquellas personas que no tengan una capacidad económica suficiente, puedan acceder a ellos. Es necesario señalar que países en la región como Argentina, que tienen un sistema normativo extenso en materia de técnicas de reproducción humana asistida, expertos del Hospital Italiano de Buenos Aires, proponen como edad límite para las donantes de útero los 50 años, y para las futuras receptoras que sean pacientes menores de 38 años, con análisis previos de buena reserva ovárica, que aseguren la posibilidad de lograr futuros embarazos; hecho que se podría tener en consideración en Colombia, si eventualmente se regula este tipo de trasplante en nuestro país.

Nos asalta una duda cuya respuesta será más compleja, pero es éste el escenario preciso para plantearla, sabedores que la respuesta solo se construirá en los próximos años, y es: luego de este avance hito en la medicina ¿cuándo se podrá realizar el trasplante de útero en mujeres transexuales?, en especial aquellas que se encuentre pasando por un proceso de reasignación de sexo y su deseo sea ser madres a través de esta práctica médico-quirúrgica. Sin duda el debate que se abordará en un futuro será agobiante, pero su viabilidad médica y anatómica está demostrada.

\section{Conclusiones}

La medicina y en especial los procedimientos médico-quirúrgicos, han desbordado el derecho positivo en los últimos 30 años en nuestro país; siendo en la actualidad el trasplante uterino el nuevo desafío de la ciencia médica, que pone en apuros al ordenamiento jurídico nacional, por la falta de regulación de esta materia en Colombia.

El código civil, máximo instrumento de codificación de las relaciones entre los particulares (artículo 1 Código Civil), fue sancionado hace más de un siglo, razón por la cual no pudo haber regulado aspectos que son de reciente desarrollo. Sin embargo, es cuestionable que el legislador actual aún, a pesar de conocer de los adelantos biotecnológicos y médicos, no se haya dedicado a dar respuesta a los problemas que estos plantean a la ciencia jurídica. (Moadie, 2011). Toda vez que las normas deben estar acorde con las circunstancias de modo, tiempo y lugar, porque este tipo de prácticas médicas como lo es el trasplante uterino, puede impactar en las finanzas del Estado colombiano.

Lo más destacable es que el gobierno nacional en los últimos meses, sancionó la Ley 1953 del 2019, que establece los lineamientos para el desarrollo de la política pública de prevención de la infertilidad y su tratamiento dentro de los parámetros de salud reproductiva, por lo cual acogió la definición emitida sobre infertilidad por la OMS, y le ordenó al Ministerio de Salud crear nuevas políticas

Página | 85 
públicas de infertilidad, con miras a garantizar el pleno ejercicio de las garantías sexuales y reproductivas de los ciudadanos del territorio nacional.

Lo que podría conllevar una posible inclusión a mediano o largo plazo, del trasplante de útero como una nueva técnica de reproducción humana asistida en Colombia, lo cual se ha propuesto y desarrollado en este artículo, toda vez que esta técnica tiene como objeto recuperar la fertilidad en la mujer, siendo la oportunidad de acceder a algún tipo de tratamiento médico a aquellas que no tienen un útero funcional, en términos para lograr un embarazo y tengan dentro de su proyecto de vida tener un hijo biológico, para no generar una especie de desigualdad en términos de acceso a los procedimientos de reproducción humana, aun cuando es el artículo 42 de la constitución nacional, quien nos brinda la posibilidad de procrear hijos científicamente.

\section{Referencias Bibliográficas}

Aibar, Laura y Martínez, Luis. (2011). Fecundación In Vitro. Servicio de Obstetricia y Ginecología. Hospital Universitario Virgen de las Nieves.

Agencia Sinc. (2018, Diciembre 5). Nace el primer bebé tras un trasplante de útero de una donante fallecida. El Espectador. Recuperado de: https://www.elespectador.com/noticias/ciencia/na ce-el-primer-bebe-tras-un-trasplante-de-utero-deuna-donante-fallecida-articulo-827435

Carmona, Francisco. (2016). Trasplante uterino. Estado actual y perspectivas futuras. Recuperado de: http://www.drfcarmona.com/trasplante-de$\underline{\text { utero/ }}$

Correa, Alejandro. (2008). Trasplante uterino, estado del arte. Revista Colombiana de Obstetricia y Ginecología, vol. $59 \mathrm{~N}^{\circ} 4$.

Código Civil Colombiano (1873).

Constitución Política Colombiana (1991).

Ciamatori, Silvia. (2016). Trasplante de útero: algunos aspectos críticos para analizar. Editorial. Recuperado
http://www.fasgo.org.ar/images/EDITORIAL TR ANSPLANTE UTERINO.pdf

Díaz-García C, Johannesson L, Enskog A, Tzakis A, Olausson M, Brännström M (2011). Uterine transplantation research: laboratory protocols for clinical application. Instituto Valenciano de Infertilidad, and b La Fe University Hospital, Valencia, Spain.

F. Zegers-Hochschild, G. D. Adamson, J. de Mouzon, O. Ishihara, R. Mansour, K. Nygren, E. Sullivan, S. van der Poel on behalf of ICMART and WHO (2010). Glosario de terminología en Técnicas de Reproducción Asistida (TRA). Versión revisada y preparada por el International CommitteforMonitoringAssistedReproductiveTec hnology (ICMART) y la Organización Mundial de la Salud (OMS). Consultado en: http://www.cnrha.msssi.gob.es/bioetica/pdf/Tecni cas_Reproduccion_Asistida TRA.pdf

Grady, Denise. (2017, diciembre 4). Una mujer con trasplante de útero da a luz por primera vez en Estados Unidos. The New York Times. Recuperado de: https://www.nytimes.com/es/2017/12/06/unamujer-con-trasplante-de-utero-da-a-luz-porprimera-vez-en-estados-unidos/

Grupo Profesionales del Área Alexandra Betancourt. (2016). Lineamientos de promoción a la donación de órganos y tejidos con fines de trasplante de la coordinación regional $\mathrm{n}^{\circ} 1$ Red de Donación y Trasplante. Secretaria de Salud de Alcaldía Mayor de Bogotá. Recuperado de:http:/www.saludcapital.gov.co/Lineamientos/ 43 SDS PSS LN 004 Promocion Donacion O rganos_Tejidos_Con fines_Trasplantes.pdf

Hospital Italiano de Buenos Aires. Trasplante Uterino. Recuperado en: https://www1.hospitalitaliano.org.ar/\#!/home/gine cologia/seccion/19929

Prats, Jaime. (2014, Octubre 4). Nace el primer niño tras un trasplante de útero. EL PAÍS. Recuperado de: https:/elpais.com/elpais/2014/10/04/ciencia/1412 $406310 \quad 537420 . \mathrm{html}$ 
Moadie, Vanina (2011). Reflexión crítica sobre el fenómeno corporal en la legislación colombiana. Tendencias actuales del Derecho Privado. Págs. 925. ISBN: 958-8687-13-1.

Moadie, Vanina (2015). Reflexión crítica sobre la fecundación post-mortem como técnica de reproducción asistida en el ordenamiento jurídico colombiano y su incidencia en el ámbito filial y sucesoral. XI Jornadas de sociología. Facultad de Ciencias Sociales, Universidad de Buenos Aires, Buenos Aires. Recuperado en: http://cdsa.aacademica.org/000-061/327.pdf

República de Colombia (2004). Decreto 2493, por el cual se reglamentan parcialmente las leyes $9^{\circ}$ de 1979 y 73 de 1988, en relación con los componentes anatómicos.

República de Colombia (2016). Ley 1805, por medio del cual se modifican la Ley 73 de 1988 y la Ley 919 de 2004, en materia de donación de componentes anatómicos y se dictan otras disposiciones.

República de Colombia (2019). Ley 1953, por medio de la cual se establecen los lineamientos para el desarrollo de la política pública de prevención de la infertilidad y su tratamiento dentro de los parámetros de salud reproductiva.

Santamaría Solís, (2000). Técnicas de reproducción asistida. Aspectos bioéticos. Disponible http://aebioetica.org/revistas/2000/1/41/37.pdf The Lance. (2014). Fertility: progress and uncertainty. Editorial, Vol. 384, p. 1237. DOI: https://doi.org/10.1016/S0140-6736(14)61750-5 